\section{Mehr melanozytäre Nävi nach Immunsuppression}

\begin{abstract}
Während einer immunsuppressiven Therapie erhöht sich die Zahl der melanozytären Nävi und verändert sich deren dermatoskopisches Bild, wie einer prospektive türkische Fall-Kontroll-Studie ergab. Ein Zusammenhang mit der Entwicklung von Melanomen ist jedoch nicht erkennbar.
\end{abstract}

$D_{\mathrm{r}}^{\mathrm{i}}$ ie plötzliche Zunahme von gutartigen Fehlbildungen der Haut und der Immunsuppression ist ein bekanntes Phänomen bei Patienten nach einer Organtransplantation, bei Aidspatienten oder bei Schwangeren. Türkische Dermatologen haben nun die Entwicklung von Nävi bei Patienten geprüft, die zur immunsuppressiven Therapie einen Anti-TNF- $\alpha$ Antikörper, Methotrexat, Azathioprin oder Ciclosporin erhalten hatten. Für ihre Studie beurteilten sie bei 103 Patienten die Entwicklung von insgesamt 266 melanozytären Läsionen. Als Kontrolle dienten 60 Gesunde mit 180 Nävi. Dabei unterschied sich die Zusammensetzung der Studiengruppen hinsichtlich Alter, Geschlecht, Sonnenbrand in der Anamnese, Dauer der Sonnenexposition und Prävention sowie des Hauttyps nach Fitzpatrick nicht. Ziel der Untersuchung war es, zu erfahren, wie sich Zahl und Morphologie unter der Therapie verändern und wie groß das Risiko ist, dass sich aus den Läsionen Melanome entwickeln.

Signifikante Unterschiede bei der Zahl der Nävi stellten die Ärzte nach einem Jahr nur bei einer Antikörpertherapie und einer Behandlung mit Methotrexat oder Azathioprin fest, Ciclosporin hatte offenbar keinen Effekt. Der Durchmesser der Nävi hatte sich nur unter der Therapie mit dem Antikörper oder mit Methotrexat signifikant vergrößert. Beim Vergleich mit der gesunden Kontrollgruppe war nur die Zahl der Nävi statistisch signifikant größer.

Morphologische Veränderungen wurden nur bei den Patienten mit immunsuppressiver Therapie beobachtet, und zwar bereits nach sechs Monaten und nach einem Jahr Behandlung. Aller- dings waren die Unterschiede im Vergleich mit den Läsionen zu Studienbeginn nicht signifikant, wohl jedoch im Vergleich mit den Nävi bei Gesunden.

Insgesamt zehn melanozytäre Läsionen wurden schließlich exzidiert, weil sich bei ihnen hohe dermatoskopische Scores ergeben hatten, beurteilt unter anderem anhand der 7-Punkte-Checkliste nach Argenziano und der ABCDRegel. Vier Läsionen wurden zu Studienbeginn und sechs Läsionen aufgrund der dermatoskopischen Veränderungen während der Therapie histopathologisch untersucht. Alle untersuchten Proben entpuppten sich als benigne.

Fazit: Immunsuppression führt zu einer Nävi-Zunahme. Die Aussagekraft der Studie ist allerdings dadurch eingeschränkt, dass das Follow-up nur ein Jahr betrug und die Zahl der Studienteilnehmer vergleichsweise gering war. $\mathrm{Zu}-$ dem blieben die jeweiligen Indikationen für die immunsuppressive Therapie unberücksichtigt.

Peter Leiner

\section{Koseoglu G et al. Dermoscopic changes in} melanocytic nevi in patients receiving immunosuppressive and biologic treatments: Results of a prospective case-control study. JAAD 2015; 73: 623-9

\title{
Gefahren des Wildpinkelns in Indien
}

Ein 46-jähriger Bauer wurde von einer Schlange in den Penis gebissen, als er auf freiem Feld sein Wasser abschlug. Drei Stunden später erreichte er eine Nothilfe. Der Patient beschrieb die Schlange als eine "Gunas". Dieser Name ist in der Gegend für die Levanteotter (Macrovipera lebetina) üblich. Die Vitalparameter

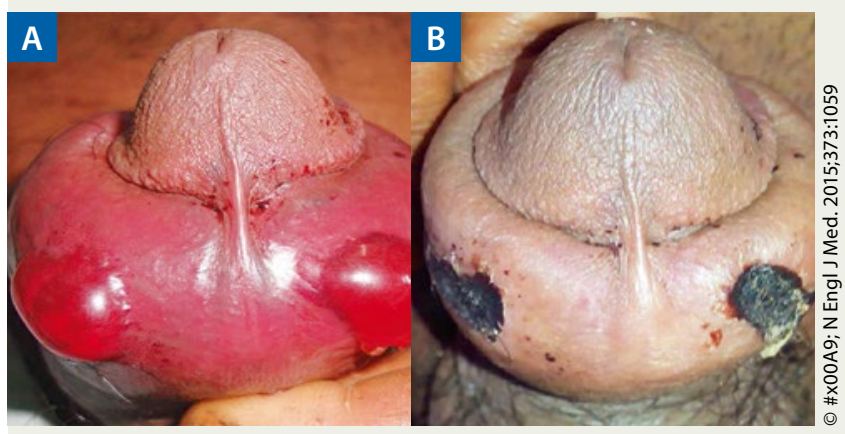

Geschwollener Penis mit Blasen an den Bissstellen (A), Zustand nach vier Tagen (B). des Patienten waren unauffällig, sein Penis war massiv geschwollen und wies zwei hämorrhagische Blasen an den Punktionsstellen der Zähne auf (Abb. A). Die Prothrombin-Zeit lag bei Aufnahme bei $17 \mathrm{~s}$ (Referenzbereich $<12$ ), die partielle Thromboplastinzeit bei $34 \mathrm{~s}$ (Referenzbereich < 24), der Fibrinogenspiegel war mit $80 \mathrm{mg} / \mathrm{dl}$ deutlich erniedrigt (Normalbereich 20-400). Die Dopplersonografie der venösen und arteriellen Gefäße des Penis war unauffällig.

Der Patient erhielt ein polyvalentes Schlangenserum, das er komplikationslos vertrug. Bei einer Nachkontrolle vier Tage nach der Entlassung war die Schwellung des Penis weitgehend zurückgegangen, an der Eintrittsstelle der Zähne hatten sich Nekrosen gebildet (Abb. B). Nach weiteren zwei Wochen waren die Verletzungen ohne Residuen abgeheilt. Leider erfahren wir nicht, in welcher Stellung der Bauer sich erleichterte, aber wahrscheinlich kauerte er sich auf den Boden. Pinkelt der Mann im Stehen, ist eine derartige Verletzung schwer vorstellbar.

Prof. Hermann S. FüeßI

Hussain T et al. A viper bite. N Engl J Med. 2015; 373: 1059 